\title{
Survival After Palliative Radiotherapy in Patients with Breast Cancer and Bone-only Metastases
}

\author{
CARSTEN NIEDER $^{1,2}$, ASTRID DALHAUG $^{1,2}$, ADAM PAWINSKI $^{1}$, \\ BÅRD MANNSÅKER ${ }^{1}$ and ELLINOR HAUKLAND ${ }^{1,2}$ \\ ${ }^{1}$ Department of Oncology and Palliative Medicine, Nordland Hospital, Bod $\phi$, Norway; \\ ${ }^{2}$ Department of Clinical Medicine, Faculty of Health Sciences, \\ UiT - The Arctic University of Norway, Troms $\phi$, Norway
}

\begin{abstract}
Background/Aim: Patients with bone-only metastases survive longer than patients with widespread visceral disease. We analyzed the prognostic impact of different baseline parameters, such as abnormal blood tests and receptor status in patients who received local radiotherapy, in addition to contemporary systemic treatment, according to national guidelines. Patients and Methods: Retrospective uni- and multivariate analyses of 57 consecutive female patients treated in the time period 2007-2014 (median follow-up=29 months). Results: The median age was 59 years and the median time interval from the initial diagnosis of breast cancer was 57 months. The median survival was 23 months from radiotherapy and 32 months from initial diagnosis of metastatic disease. Fiveyear survival rates were 13 and $21 \%$, respectively. Survival after radiotherapy was significantly longer in patients who were prescribed higher radiation doses; 29 months after $\geq 30$ Gy and 10 months after $<30 \mathrm{~Gy}, p=0.02$. Multivariate analysis confirmed 4 independent prognostic factors for shorter survival: triple-negative histology $(p=0.0001)$, high serum lactate dehydrogenase $(L D H) \quad(p=0.001)$, high serum alkaline phosphatase $(A L P)(p=0.015)$ and intended radiation dose $<30$ $G y(p=0.028)$. A 3-tiered prognostic score with median survival of 48, 21 and 12 months was developed. Conclusion: Prognosis varied widely with many patients surviving for several years. The results of simple blood tests provided important prognostic information. Prospective studies are necessary to confirm that more aggressive radiotherapy improves survival in patients with bone-only disease suitable for local therapy.
\end{abstract}

This article is freely accessible online.

Correspondence to: Carsten Nieder, MD, Department of Oncology and Palliative Medicine, Nordland Hospital, 8092 Bod $\varnothing$, Norway, Tel: +47 75578449, Fax: +47 75534975, e-mail: carsten.nieder@nlsh.no

Key Words: Bone metastases, breast cancer, prognostic factors, radiotherapy, biomarkers.
Patients with metastatic breast cancer often have widespread disease in various locations, such as lungs, pleura, liver, lymph nodes and bones (1). Relatively few patients present with limited organ involvement, e.g. brain-only or bone-only disease $(2,3)$. Use of radiotherapy is common in this subgroup $(4,5)$. While the chance of long-term survival is higher in patients with smaller tumor burden, large individual variation exists (6). Performance status, organ function, biological disease characteristics and eligibility for effective local and systemic treatment are important prognostic and predictive factors (7). Prognostic models might be helpful in pre-treatment assessment. In this regard, patients with bone-only metastases have not been studied extensively. Therefore, we evaluated the survival and prognostic factors, including a panel of blood tests, in patients treated with radiotherapy for bone-only metastases.

\section{Patients and Methods}

Patients and treatment. This retrospective intention-to-treat study included 57 consecutive female patients with bone metastases from breast cancer who received palliative external beam radiotherapy at Nordland Hospital Bodø, Norway (2-D or 3-D treatment planning, no stereotactic ablative radiotherapy). All patients were treated between 2007 and 2014 and identified from an electronic database (ARIA; Varian Medical Systems, Inc., Palo Alto, CA, USA). Some patients presented with bone metastases at first cancer diagnosis, others later during the disease trajectory; all had histological confirmation of malignancy. Staging included computed tomography of the thorax, abdomen and pelvis and radioisotope bone scan, supplemented by magnetic resonance imaging and/or ultrasound to clarify equivocal findings. If necessary to confirm the diagnosis of metastatic disease, bone biopsy was performed. Systemic treatment was given according to the guidelines of the Norwegian Breast Cancer Group (NBCG), which are stratified by biological subtype and available online (www.nbcg.no). Nationwide adherence to these guidelines is excellent. Radiotherapy fractionation was at the discretion of the treating oncologist. Radionuclide treatment was not available.

Blood tests. Lactate dehydrogenase (LDH), albumin, hemoglobin, C-reactive protein (CRP), calcium, creatinine and alkaline phosphatase (ALP) were part of routine blood chemistry and 
imaging assessment in patients with metastatic breast cancer However, some patients had missing values for one or two of these analyses. Tumor marker analyses were left to the discretion of the treating oncologist. The hospital's electronic patient record system DIPS (DIPS ASA, Bodø, Norway) was used to collect all baseline data, including blood tests. The latter had to be no older than 2 weeks before the first fraction of radiotherapy. Elevated LDH was defined as $\geq 205 \mathrm{U} / 1$ according to the hospital's reference value (low albumin $<34 \mathrm{~g} / \mathrm{l}$; high ALP $\geq 105 \mathrm{U} / \mathrm{l}$; normal CRP $<5 \mathrm{mg} / \mathrm{l}$; low hemoglobin $<11.7 \mathrm{~g} / \mathrm{dl}$; high calcium $>2.55 \mathrm{mmol} / \mathrm{l}$; low creatinine $<45 \mu \mathrm{mol} / 1)$. Low creatinine was included because it might indicate low muscle mass and impaired nutrition. Normal cancer antigen (CA) 15-3 was defined as 0-25 kIE/l.

Statistical methods. All analyses were performed with SPSS 22 (IBM, New York, NY, USA). Actuarial survival from the first day of radiotherapy was calculated with the Kaplan-Meier method and compared between different groups with the log-rank test. Multivariate analysis consisted of Cox regression (forward stepwise method). A $p$-value $\leq 0.05$ was considered statistically significant. Sixteen patients were alive at last follow-up (November 01, 2015) with a median follow-up of 29 months. Date of death was known in all other patients. As a retrospective quality of care analysis, no approval from the Regional Committee for Medical and Health Research Ethics (REK Nord) was necessary. This research project was carried out according to our Institution's guidelines and with permission to access the patients' data.

\section{Results}

Patients' characteristics. Five patients (9\%) had no previous history of cancer, i.e. bone metastases at initial diagnosis or de novo stage IV disease, and 52 developed metastases at later time points. The median age was 59 years (range $=33$ 86). The median time interval from the initial diagnosis of breast cancer was 57 months (range=1-348) and the median time interval from metastatic disease to radiotherapy was 3 months (range=1-72). Patients' characteristics are shown in Table I. The most common fractionation regimen was 10 fractions of 3 Gy (56\%) followed by 5 fractions of 4 Gy $(18 \%)$. Sixteen percent received a single fraction of $8 \mathrm{~Gy}$ and 9\% doses higher than 30 Gy. Thirty patients $(53 \%)$ had spinal target volumes. Thirty-four patients $(60 \%)$ received radiotherapy to one target volume and $40 \%$ to, at least, two target volumes during the same treatment course. All but one patient completed their prescribed course of radiotherapy.

Overall survival. The median survival was 23 months from radiotherapy and 32 months from initial diagnosis of metastatic disease. Five-year survival rates were 13 and $21 \%$, respectively (Figures 1 and 2). Survival after radiotherapy was significantly longer in patients who were prescribed higher radiation doses; 29 months after $\geq 30$ Gy and 10 months after $<30 \mathrm{~Gy}, p=0.02$. As shown in Table I, a large number of other patient-, disease- and treatment-related baseline characteristics were associated with survival after radiotherapy. These included, for example, triple-negative breast cancer histology, LDH, albumin, ALP and performance status. All parameters with a $p$-value $<0.1$ in univariate log-rank analyses were included in a multivariate regression model. The latter confirmed 4 independent prognostic factors for shorter survival: triple-negative histology $(p=0.0001)$, high LDH $(p=0.001)$, high ALP $(p=0.015)$ and intended radiation dose $<30$ Gy $(p=0.028)$.

\section{Discussion}

Patients with metastatic breast cancer often present with bone metastases or develop skeletal involvement during the disease trajectory (8). A Danish study reported by Jensen et al. estimated the overall and annual incidence of bone metastases and skeletal-related events (SRE) in newly-diagnosed breast cancer patients (1999 to 2007) using the National Patient Registry (9). Of the 35,912 patients, $178(0.5 \%)$ presented with bone metastases at the time of primary breast cancer diagnosis and, of these, $43 \%$ developed an SRE during follow-up. The term SRE relates to pathological fractures, orthopedic surgery, spinal cord compression and utilization of radiotherapy. A total of 1,272 of 35,690 (3.6\%) patients without bone metastases at diagnosis developed bone metastases during a median followup time of 3.4 years. Among these patients, $46 \%$ subsequently developed an SRE. The 5-year survival rate of Danish patients with bone metastases was $8 \%(10)$.

A limited number of studies have focused on patients with bone-only metastases. Turanli and Cetin reported a cohort of 129 patients treated between 2004 and 2007 (11). Only 24 patients received palliative radiotherapy. A limited number of baseline characteristics were reported and analyzed as potential prognostic factors for improved survival. Two of these were statistically significant: normal serum CA 15-3 level and postmenopausal status. Hormone receptor and HER2 status were not significant. Blood tests other than CA 15-3 were not included. In a later article, this group reported on 101 postmenopausal hormone receptor-positive patients, treated between 2001 and 2007, who were classified into two groups according to initial treatment modalities; patients who received endocrine therapy only (group I) and chemotherapy followed by endocrine therapy (group II) (12). All patients received bisphosphonates, whereas only 24 patients required palliative radiotherapy during the course of their disease. In groups I and II, the median time to progression was 12 and 16 months $(p=0.96)$ and median overall survival 41 and 40 months $(p=0.79)$, respectively. In a different study, median survival after bone metastases diagnosis was 28 months in women with bone-only metastases (13), largely comparable to the present data (32 months).

Our study shares limitations with previous ones, e.g. the retrospective study design. Only 57 patients could be included, resulting in small subgroups and limited statistical power. A 
Table I. Patients' characteristics before radiotherapy (RT) and survival outcomes ( $n=57)$.

\begin{tabular}{|c|c|c|c|c|}
\hline Parameter & $\mathrm{n}$ & $\%$ & Median survival, months & $p$-Value \\
\hline Age $<60$ years & 30 & 53 & 17 & \\
\hline Age $\geq 60$ years & 27 & 47 & 29 & 0.06 \\
\hline ER- and/or PR-positive & 47 & 82 & 27 & \\
\hline ER- and PR-negative & 10 & 18 & 15 & 0.27 \\
\hline HER2-positive ${ }^{1}$ & 9 & 17 & 42 & \\
\hline HER2-negative ${ }^{1}$ & 45 & 83 & 21 & 0.25 \\
\hline Triple-negative $^{1}$ & 8 & 15 & 14 & \\
\hline Other than triple-negative ${ }^{1}$ & 46 & 85 & 27 & 0.01 \\
\hline Metastases at first diagnosis & 5 & 9 & Not reached & \\
\hline Metachronous metastatic disease & 52 & 91 & 21 & 0.27 \\
\hline Solitary bone metastasis & 23 & 40 & 42 & \\
\hline Multiple bone metastases & 34 & 60 & 17 & 0.05 \\
\hline ECOG PS 0 & 23 & 40 & 32 & \\
\hline ECOG PS 1 & 17 & 30 & 26 & \\
\hline ECOG PS 2 & 11 & 19 & 17 & \\
\hline ECOG PS 3 & 6 & 11 & 5 & $0.01^{2}$ \\
\hline Spinal target volume & 30 & 53 & 20 & \\
\hline No spinal target volume & 27 & 47 & 23 & 0.75 \\
\hline Metastatic spinal cord compression & 7 & 12 & 16 & \\
\hline No metastatic spinal cord compression & 50 & 88 & 26 & 0.99 \\
\hline One target volume & 34 & 60 & 30 & \\
\hline At least 2 target volumes & 23 & 40 & 15 & 0.03 \\
\hline Serum albumin normal ${ }^{1}$ & 51 & 93 & 27 & \\
\hline Serum albumin low 1 & 4 & 7 & 7 & 0.02 \\
\hline Serum LDH normal ${ }^{1}$ & 29 & 58 & 41 & \\
\hline Serum LDH high ${ }^{1}$ & 21 & 42 & 13 & 0.0001 \\
\hline Serum ALP normal ${ }^{1}$ & 29 & 58 & 31 & \\
\hline Serum ALP high ${ }^{1}$ & 21 & 42 & 15 & 0.002 \\
\hline Serum calcium normal ${ }^{1}$ & 49 & 89 & 27 & \\
\hline Serum calcium high ${ }^{1}$ & 6 & 11 & 16 & 0.67 \\
\hline Serum CRP normal ${ }^{1}$ & 37 & 67 & 29 & \\
\hline Serum CRP high ${ }^{1}$ & 18 & 33 & 13 & 0.02 \\
\hline Serum creatinine normal or high ${ }^{1}$ & 51 & 91 & 27 & \\
\hline Serum creatinine low 1 & 5 & 9 & 8 & 0.01 \\
\hline Serum hemoglobin normal ${ }^{1}$ & 45 & 80 & 26 & \\
\hline Serum hemoglobin low 1 & 11 & 20 & 10 & 0.08 \\
\hline Serum CA 15-3 normal ${ }^{1}$ & 10 & 22 & 41 & \\
\hline Serum CA 15-3 high ${ }^{1}$ & 35 & 78 & 26 & 0.06 \\
\hline Opioid analgesics 1 & 36 & 68 & 20 & \\
\hline No opioid analgesics 1 & 17 & 32 & 26 & 0.40 \\
\hline Charlson comorbidity index 0 & 35 & 61 & 23 & \\
\hline Charlson comorbidity index $\geq 1$ & 22 & 39 & 21 & 0.21 \\
\hline
\end{tabular}

${ }^{1}$ Information not available in all patients. ${ }^{2} p$-value calculated over all strata. ER, Estrogen receptor; PR, progesterone receptor; ECOG PS, Eastern Cooperative Oncology Group performance status; LDH, lactate dehydrogenase; ALP, alkaline phosphatase; CRP, C-reactive protein; CA, cancer antigen.

typical patient in our study was a postmenopausal woman with estrogen receptor-positive and HER2-negative disease and good performance status who developed metachronous metastases (Table I). According to the national guidelines, such patients receive zoledronic acid and several lines of sequential hormonal and cytotoxic therapy, depending on tumor load, response to previous therapy and other parameters. We based our survival analyses on the start of radiotherapy rather than diagnosis of metastases because fewer patients had available baseline blood tests at this earlier point in time. In other words, we prioritized group size and completeness of data. As a drawback, blood test results were affected by ongoing systemic treatment. Given that most patients received early radiotherapy, we believe that largely comparable results may be expected in either scenario. Patterns of progression and cause of death were not evaluated. 


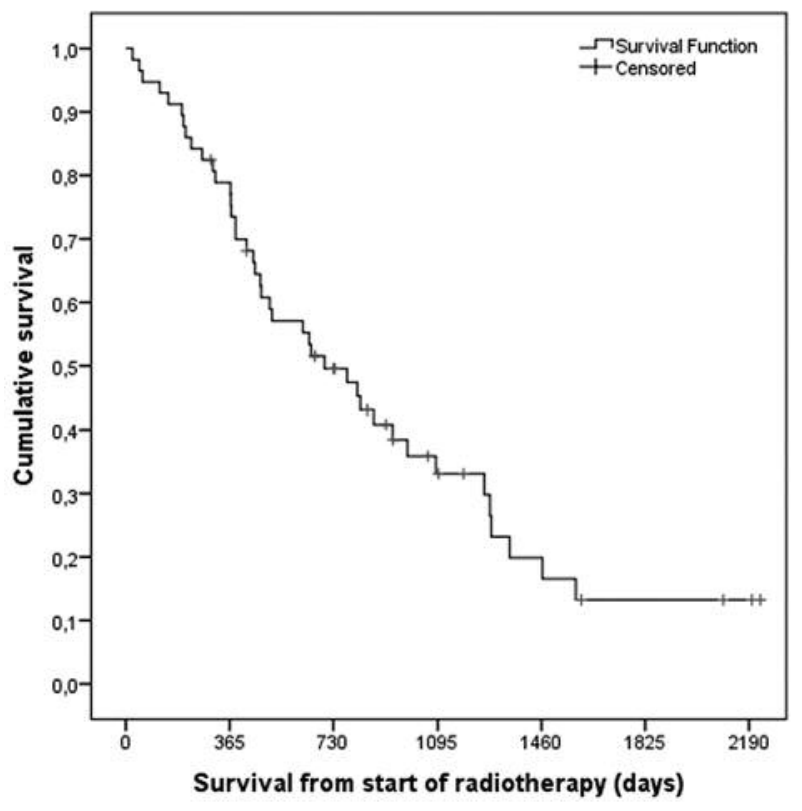

Figure 1. Actuarial Kaplan-Meier survival curve for 57 patients with bone-only metastases from breast cancer, calculated from the start of radiotherapy.

We found several disease characteristics to be associated with survival after radiotherapy. These included, for example, breast cancer type (shortest survival for triple-negative status; median=14 months), LDH and ALP, but not age. Interestingly, prescription of higher radiation doses was associated with improved survival in multivariate analysis. It has long been hypothesized that patients with oligometastases may benefit from more aggressive local therapy $(14,15)$. However, only randomized trials can provide high level evidence for or against combined modality treatment. In the present study, different sources of bias may have been involved as radiation dose was at the discretion of the treating physician. Experienced oncologists are likely to identify patients with favorable prognosis (16). Subtle imbalance in baseline characteristics and radiation field sizes/expected bone marrow compromise, which were not captured in the multivariate analysis, could be responsible for the outcomes observed. Therefore, we decided to develop a preliminary prognostic model based on the remaining three parameters, which are objectively measurable. Figure 3 shows the survival outcomes for patients in the respective risk groups (median=48, 21 and 12 months, $p=0.0001$ ). This model should be validated in larger studies and may afterwards be used for stratification when performing prospective studies of different radiotherapy regimens.

A drawback of most studies, resulting in difficulties with inter-study comparisons, is that not all potential prognostic

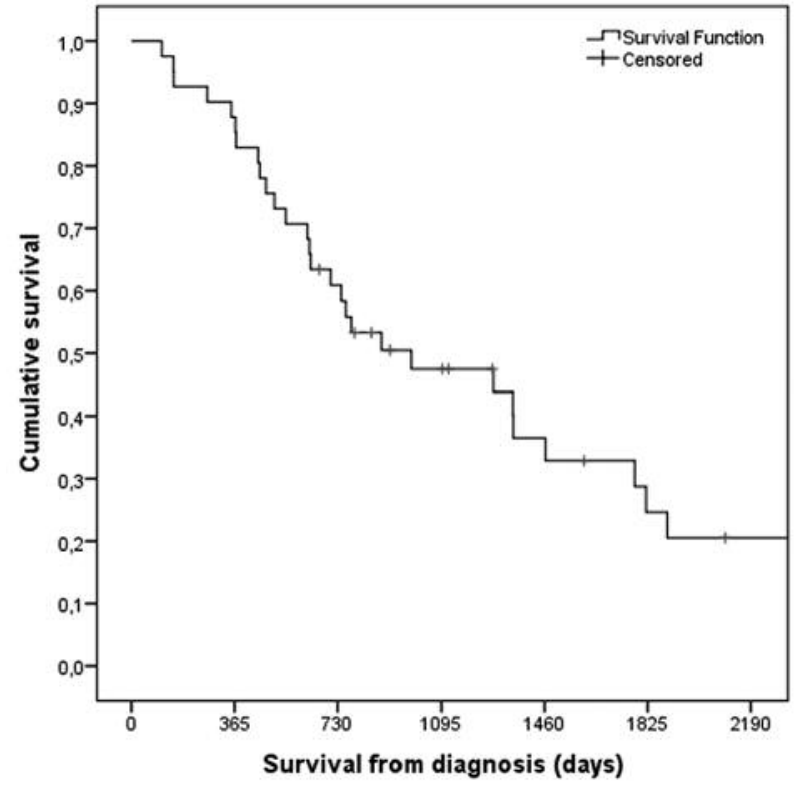

Figure 2. Actuarial Kaplan-Meier survival curve for 57 patients with bone-only metastases from breast cancer, calculated from the diagnosis of metastatic disease.

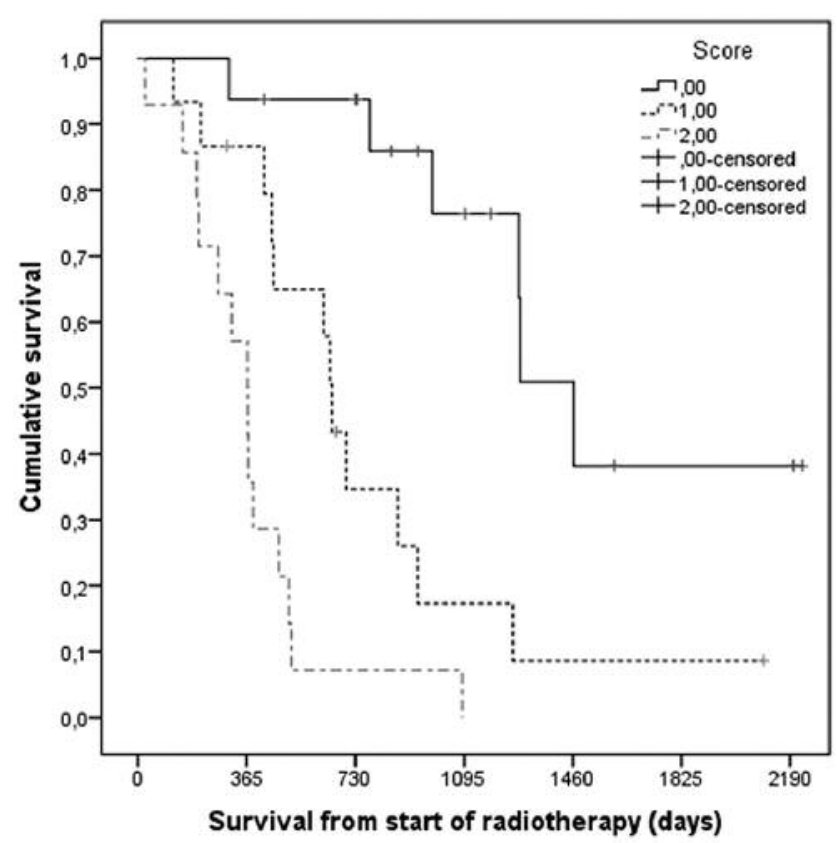

Figure 3. Actuarial Kaplan-Meier survival curves for patients with bone-only metastases from breast cancer stratified by risk score. Median survival was 48 months in patients without risk factors (normal lactate dehydrogenase $(L D H)$ and alkaline phosphatase (ALP), not triplenegative, $n=16), 21$ months in patients with one risk factor $(n=15)$ and 12 months in patients with more than one risk factor $(n=14), p=0.0001$ (log-rank test over all three strata). 
factors were included, e.g. LDH and ALP. Emerging prognosticators, such as circulating tumor cells have not yet been studied in the present setting (17). These considerations are also true for a relatively large series from the M.D. Anderson Cancer Center (18). The analysis included 314 patients managed between 1997 and 2008. A considerable proportion of all patients with bone metastases had boneonly disease $(33.5 \%)$. The multivariate analysis of the latter group showed that survival was influenced by the presence of solitary bone metastases and absence of bone pain.

\section{Conclusion}

Prognosis was highly variable but long-term survival was not uncommon. Especially patients with receptor-positive disease and normal LDH and ALP survived for several years. The results of simple blood tests provided important prognostic information. Prospective studies are necessary to confirm that more aggressive radiotherapy improves survival in patients with bone-only disease suitable for local therapy.

\section{References}

1 Kimbung S, Loman N and Hedenfalk I: Clinical and molecular complexity of breast cancer metastases. Semin Cancer Biol 35: 85-95, 2015

2 James JJ, Evans AJ, Pinder SE, Gutteridge E, Cheung KL, Chan $\mathrm{S}$ and Robertson JF: Bone metastases from breast carcinoma: histopathological - radiological correlations and prognostic features. Br J Cancer 89: 660-665, 2003

3 Nieder C, Oehlke O, Hintz M and Grosu AL: The challenge of durable brain control in patients with brain-only metastases from breast cancer. Springerplus 4: 585, 2015.

4 Steinauer K, Gross MW, Huang DJ, Eppenberger-Castori S and Güth U: Radiotherapy in patients with distant metastatic breast cancer. Radiat Oncol 9: 126, 2014.

5 Feyer PC and Steingraeber M: Radiotherapy of bone metastasis in breast cancer patients - Current approaches. Breast Care (Basel) 7: 108-112, 2012.

6 Weide R, Feiten S, Friesenhahn V, Heymanns J, Kleboth K, Thomalla J, van Roye $\mathrm{C}$ and Köppler H: Metastatic breast cancer: Prolongation of survival in routine care is restricted to hormonereceptor- and Her2-positive tumors. Springerplus 3: 535, 2014.

7 Bidard FC, Peeters DJ, Fehm T, Bidard FC, Peeters DJ, Fehm T, Nolé F, Gisbert-Criado R, Mavroudis D, Grisanti S, Generali D, Garcia-Saenz JA, Stebbing J, Caldas C, Gazzaniga P, Manso L, Zamarchi R, de Lascoiti AF, De Mattos-Arruda L, Ignatiadis M, Lebofsky R, van Laere SJ, Meier-Stiegen F, Sandri MT, Vidal-Martinez J, Politaki E, Consoli F, Bottini A, Diaz-Rubio E, Krell J, Dawson SJ, Raimondi C, Rutten A, Janni W, Munzone E, Carañana V, Agelaki S, Almici C, Dirix L, Solomayer EF, Zorzino L, Johannes H, Reis-Filho JS, Pantel K, Pierga JY and Michiels S: Clinical validity of circulating tumour cells in patients with metastatic breast cancer: A pooled analysis of individual patient data. Lancet Oncol 15: 406-414, 2014.
8 Li BT, Wong MH and Pavlakis N: Treatment and prevention of bone metastases from breast cancer: A comprehensive review of evidence for clinical practice. J Clin Med 3: 1-24, 2014.

9 Jensen AØ, Jacobsen JB, Nørgaard M, Yong M, Fryzek JP and Sørensen HT: Incidence of bone metastases and skeletal-related events in breast cancer patients: a population-based cohort study in Denmark. BMC Cancer 11: 29, 2011.

10 Yong M, Jensen AÖ, Jacobsen JB, Nørgaard M, Fryzek JP and Sørensen HT: Survival in breast cancer patients with bone metastases and skeletal-related events: a population-based cohort study in Denmark (1999-2007). Breast Cancer Res Treat 129: 495-503, 2011.

11 Turanli S and Cetin A: Prognostic role of serum cancer antigen 15-3 in breast cancer patients with isolated bone metastases. Biomarkers 15: 418-423, 2010.

12 Turanli S, Oksuzoglu B, Bulak H and Cetin A: What is the best treatment option in postmenopausal, hormone responsive breast cancer patients with isolated bone metastases? Indian J Cancer 50: 52-57, 2013.

13 Harries M, Taylor A, Holmberg L, Agbaje O, Garmo H, Kabilan $\mathrm{S}$ and Purushotham $\mathrm{A}$ : Incidence of bone metastases and survival after a diagnosis of bone metastases in breast cancer patients. Cancer Epidemiol 38: 427-434, 2014.

14 Salama JK and Chmura SJ: The role of surgery and ablative radiotherapy in oligometastatic breast cancer. Semin Oncol 41: 790-797, 2014.

15 Di Lascio S and Pagani O: Oligometastatic breast cancer: A shift from palliative to potentially curative treatment? Breast Care (Basel) 9: 7-14, 2014.

16 Kiely BE, Martin AJ, Tattersall MH, Nowak AK, Goldstein D, Wilcken NR, Wyld DK, Abdi EA, Glasgow A, Beale PJ, Jefford M, Glare PA and Stockler MR: The median informs the message: Accuracy of individualized scenarios for survival time based on oncologists' estimates. J Clin Oncol 31: 3565-3371, 2013.

17 Ravelli A, Reuben JM, Lanza F, Anfossi S, Cappelletti MR, Zanotti L, Gobbi A, Senti C, Brambilla P, Milani M, Spada D, Pedrazzoli P, Martino M, Bottini A and Generali D; Solid Tumor Working Party of European Blood and Marrow Transplantation Society (EBMT): Breast cancer circulating biomarkers: advantages, drawbacks, and new insights. Tumour Biol 36: 6653-6665, 2015.

18 Niikura N, Liu J, Hayashi N, Palla SL, Tokuda Y, Hortobagyi GN, Ueno NT and Theriault RL: Retrospective analysis of antitumor effects of zoledronic acid in breast cancer patients with bone-only metastases. Cancer 118: 2039-2047, 2012.
Received August 19, 2016

Revised August 29, 2016

Accepted August 30, 2016 